\title{
Trade-Off between Information Format and Capacity in the Olfactory System
}

\author{
(D)Zane N. Aldworth and ${ }^{-M a r k}$ A. Stopfer \\ NIH-NICHD, Laboratory of Cellular and Synaptic Physiology, Bethesda, Maryland 20892
}

\begin{abstract}
As information about the sensory environment passes between layers within the nervous system, the format of the information often changes. To examine how information format affects the capacity of neurons to represent stimuli, we measured the rate of information transmission in olfactory neurons in intact, awake locusts (Schistocerca americana) while pharmacologically manipulating patterns of correlated neuronal activity. Blocking the periodic inhibition underlying odor-elicited neural oscillatory synchronization increased information transmission rates. This suggests oscillatory synchrony, which serves other information processing roles, comes at a cost to the speed with which neurons can transmit information. Our results provide an example of a trade-off between benefits and costs in neural information processing.
\end{abstract}

Key words: information theory; neural coding; oscillations; synchrony

\section{Introduction}

The brain's internal representation of the environment is built from patterns of neural activity bearing information about sensory stimuli. As this activity travels through the brain, a succession of neural circuits manipulates it into a series of coding formats, each thought to provide specific advantages for processing information. How the benefits of a given format are balanced against its costs is largely unknown.

One common coding format uses periodic inhibition to coordinate neural spiking into synchronous oscillations. Oscillatory synchrony has been proposed to offer several benefits, including enhancing the discriminability of sensory representations (Stopfer et al., 1997; Perez-Orive et al., 2004) and "binding" diverse stimulus features into coherent percepts (Gray, 1999; Singer, 1999). Here we examined how neural oscillatory synchronization affects another measure of coding quality, the rate information is transmitted.

In insects, information about odors is detected by olfactory receptor neurons (Fig. 1a) and is carried to the antennal lobe (analogous to the vertebrate olfactory bulb) as excitatory input to local neurons (LNs; analogous to vertebrate granule cells), and projection neurons (PNs; analogous to vertebrate mitral and tufted cells; Seki et al., 2010; Reisenman et al., 2011). The receptor neurons, LNs, and PNs, together generate patterns of spiking in PNs that bear information about the identity, concentration, and timing of odors (Laurent and Davidowitz, 1994; Stopfer et al.,

Received Aug. 25, 2014; revised 0ct. 31, 2014; accepted Dec. 3, 2014.

Author contributions: Z.N.A. and M.A.S. designed research; Z.N.A. performed research; M.A.S. contributed unpublished reagents/analytic tools; Z.N.A. analyzed data; Z.N.A. and M.A.S. wrote the paper.

This work was supported by an intramural grant from the National Institute of Child Health and Human Development-National Institutes of Health (M.A.S.). We thank the members of the Stopfer laboratory for helpful discussions during the development of this project and Kui Sun for excellent animal care.

The authors declare no competing financial interests.

Correspondence should be addressed to Zane N. Aldworth, NIH-NICHD, Laboratory of Cellular and Synaptic Physiology, Bethesda, MD 20892. E-mail: zane.aldworth@nih.gov.

DOI:10.1523/JNEUROSCI.3562-14.2015

Copyright $\odot 2015$ the authors $\quad 0270-6474 / 15 / 351521-09 \$ 15.00 / 0$
2003; Brown et al., 2005; Mazor and Laurent, 2005). Further, LNs provide rhythmic pulses of inhibition to PNs, causing them to synchronize their firing. The resulting waves of spikes carry olfactory information to the mushroom bodies and the lateral horn (MacLeod and Laurent, 1996; Stopfer et al., 1997; Perez-Orive et al., 2002; Ito et al., 2009; Tanaka et al., 2009; Gupta and Stopfer, 2012).

Disrupting the synchronization of PNs has been shown to disrupt the ability of downstream neurons in the mushroom body (Kenyon cells and lobe neurons; Fig. 1a) to discriminate similar odorants (MacLeod et al., 1998; Perez-Orive et al., 2004), and of animals to perform difficult olfactory discrimination tasks (Stopfer et al., 1997; Hosler et al., 2000). Thus, synchronizing olfactory neurons can enhance information processing. However, because synchronization is established by imposing periodic inhibition onto PNs, it may reduce the number of spikes a PN can fire, and also constrain the variety of information-bearing patterns of spikes any single neuron can produce. Therefore, oscillatory synchronization may "buy" the benefits of synchronization from the brain's ability to more rapidly represent the external environment.

We evaluated this potential trade-off between coding format and information rate in the olfactory system of the locust. We delivered odorants as controlled, repeatable plumes and measured the ability of PNs to transmit information about the plume's temporal structure. Our results show that pharmacologically blocking synchronization led to a significant increase in information rate, suggesting that the use of a synchronous coding scheme introduces a trade-off: although synchrony allows fine discrimination, by reducing the number of spikes available for encoding information, it also reduces the ability of the system to rapidly transmit information about the stimulus.

\section{Materials and Methods}

Dissection and electrophysiology. Locusts (152) of either sex from our crowded colony were prepared for electrophysiological recording as reported previously (Brown et al., 2005). Briefly, the legs, wings, and gut were removed; the animal was waxed into place; and the brain was ex- 
posed, desheathed, and perfused with physiological saline. Recordings were made from one or several PNs either intracellularly using patch-clamp pipettes filled with locust internal solution (Laurent et al., 1993), or extracellular tetrodes (A16 A2x2-tet-3 mm-150-150-121; NeuroNexus Technologies). Spikes recorded extracellularly were sorted by a whole waveform algorithm (Pouzat et al., 2002) implemented in MATLAB (The MathWorks). Isolated extracellular units were included in our study only if they were estimated to have $<5 \%$ false positives and $5 \%$ false negatives for the duration of the recordings (Hill et al., 2011). Odor concentration was monitored by a miniature photo-ionization detector (PID; Model 200A; Aurora Scientific). Probability values of finding PID peaks during the time the odor valve was open (shifted by $50 \mathrm{~ms}$ to allow the odor to reach the antenna) were estimated from 30 trials each in 10 different experiments using the binomfit function in MATLAB. Direct afferent input to the PNs was monitored by electro-antennogram (EAG) recordings, for which chloride silver wires were gently inserted into the cut distal end of the stimulated antenna and amplified using an in-line DC amplifier (Brownlee Precision). To assess the role of fast (GABA-type) inhibition, recordings were made before and after local microinjection of the $\mathrm{GABA}_{\mathrm{a}}$ blocker picrotoxin (PCT; $\sim 5 \mathrm{pl}$ of $0.1 \mathrm{~mm}$ PCT) into the antennal lobe ( $N=19$ animals). Nonspecific effects of microinjection were evaluated by substituting insect saline for PCT ( $N=8$ animals). To monitor the effect of PCT or saline injection on odor-elicited oscillations, we recorded the local field potential with blunt glass microelectrodes placed near the output site of the PNs in the ipsilateral calyx of the mushroom body.

Odor stimulation. The olfactory system was stimulated by injecting odorized air $(0.2 \mathrm{~L} / \mathrm{min}$ of 10:1 dilution of 1-hexanol, hexanal, cyclohexanol, or 1-octanol in mineral oil) into a stream of dried, carbon-filtered air $(1.0 \mathrm{~L} / \mathrm{min})$ that was aimed at the ipsilateral intact antenna of the locust. Pulse trains of odorized air were delivered to approximate the temporal distribution of odor filaments originating from point sources in the natural environment. We constructed odor plumes by drawing the temporal length of odor filaments (burst length) and the time between onset of consecutive odor filaments (burst return) from gamma distributions using the following parameters (burst length: $k=1.3 ; \theta=294.0 \mathrm{~ms}$; burst return period: $k=2.4 ; \theta=307.7 \mathrm{~ms}$; Murlis et al., 2000) and explicitly ensuring that the burst length was always at least 20 ms shorter than the concurrent burst return period. The burst length was used to determine the period of time that a pneumatic PicoPump (WPI) valve was open, allowing the injection of odorized air into the clean air stream. The burst return period determined the length of time between consecutive openings of the valve. Odor pulse trains were presented as either a $30 \mathrm{~s}$ segment repeated up to 500 times, or as a single nonrepeating $1800 \mathrm{~s}$ segment.

Analysis. All analyses were performed using custom routines written in MATLAB (The MathWorks). All data used for hypothesis testing were examined for normality with the Lilliefors test (MATLAB's "lillietest" function). For normally distributed data, we used two-tailed paired $t$ tests
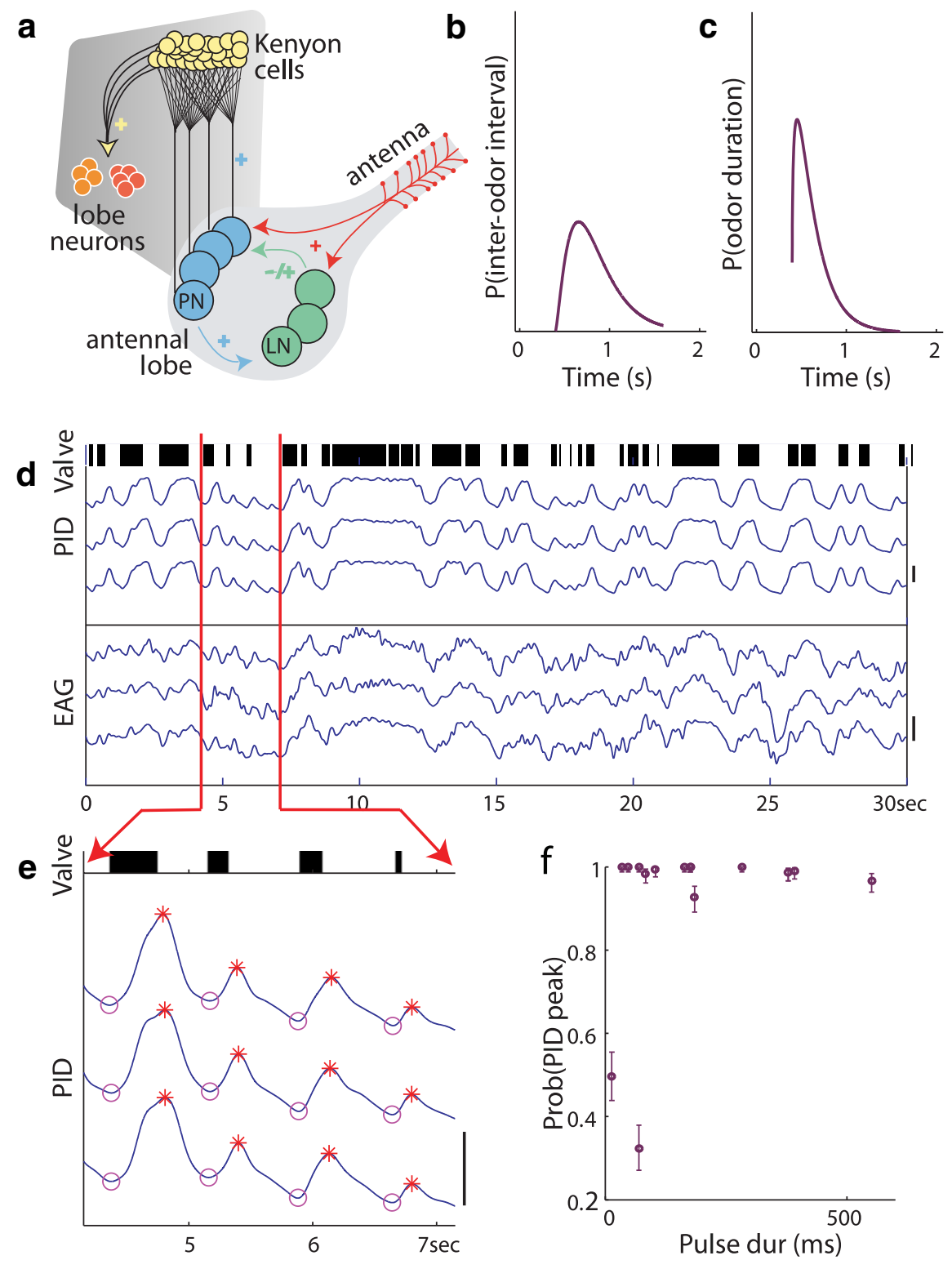

Figure 1. Dynamic odor stimulation. $\boldsymbol{a}$, Locust olfactory system. Olfactory receptor neurons in the antenna make excitatory synapses onto two populations of neurons in the antennal lobe, PNs and LNs. GABA ${ }_{a}$-mediated inhibition from LNs synchronizes the PN population, whose spikes are carried to higher order neurons including Kenyon cells. $\boldsymbol{b}, \boldsymbol{c}$, Synthetic plume was constructed from gamma distributions describing the odor burst return period $(\boldsymbol{b})$ and burst length of natural odor plumes (c). $\boldsymbol{d}$, Plume stimulus and response. Valve, odor-valve openings (black) and closings (white); PID, three example trials showing output of PID near the animal's antenna; $E A G$, same three trials showing the EAG, reflecting the antenna's summed afferent drive. $\boldsymbol{e}$, Enlarged timescale from $\boldsymbol{d}$ showing PID responses to odor-valve openings of $392,176,164$, and $35 \mathrm{~ms}$, left to right. Detected peaks are marked with red asterisks, intervening minima with magenta circles. $f$, Valve openings precede PID peaks; graph shows binomial estimation plus $95 \%$ confidence interval for finding a PID peak following isolated valve openings of varying duration (Dur). Calibration: $700 \mathrm{mV}$ (PID), $50 \mathrm{mV}$ (EAG).

using the MATLAB "ttest2" function. For other data we used either MannWhitney or Kruskal-Wallace tests, as appropriate.

The information content of neurons was estimated by the mutual information rate (Shannon, 1948) between the temporal dynamics of the odorant stimulus and the neuronal response. This was done through the "direct method" approach (Strong et al., 1998) by finding the difference between the unconditional and stimulus-conditioned response entropies:

$$
I(S ; R)=H(R)-H(R \mid S)
$$

where $R$ represents neural response, $S$ represents stimulus, and the entropy of a quantity $H(*)$ is defined as follows: 


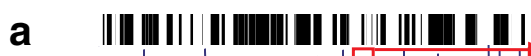

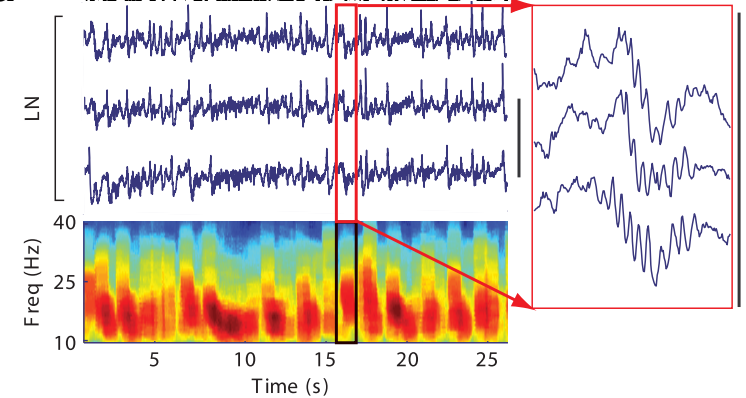

b
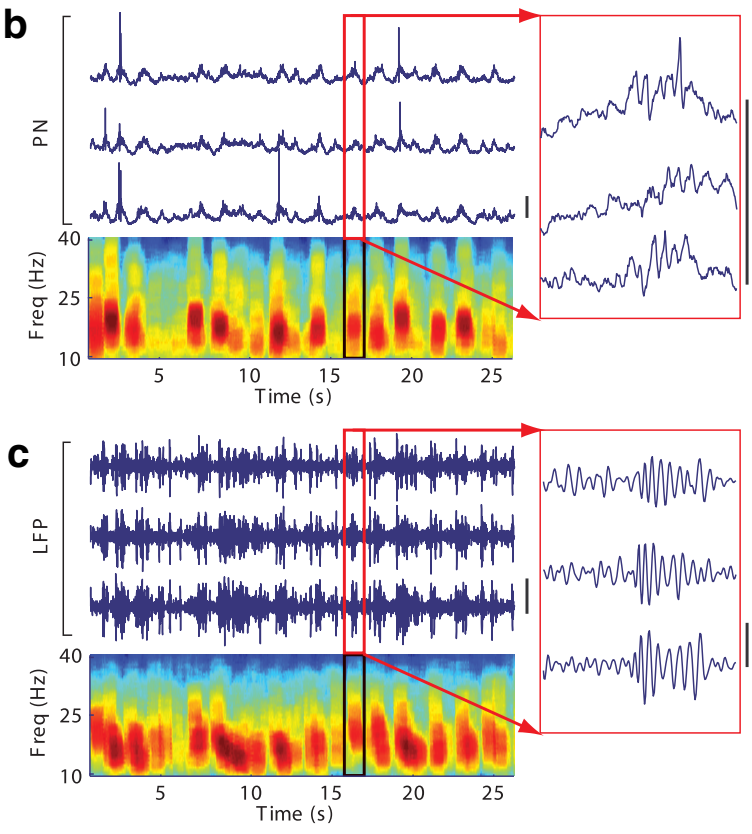

d

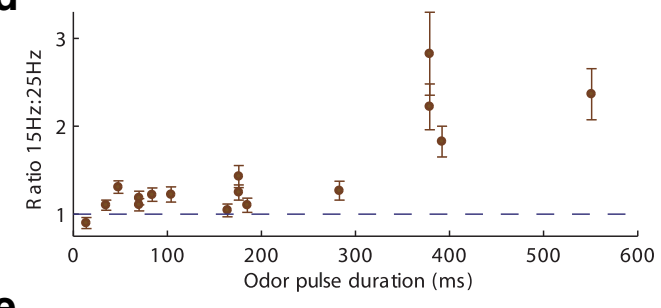

e
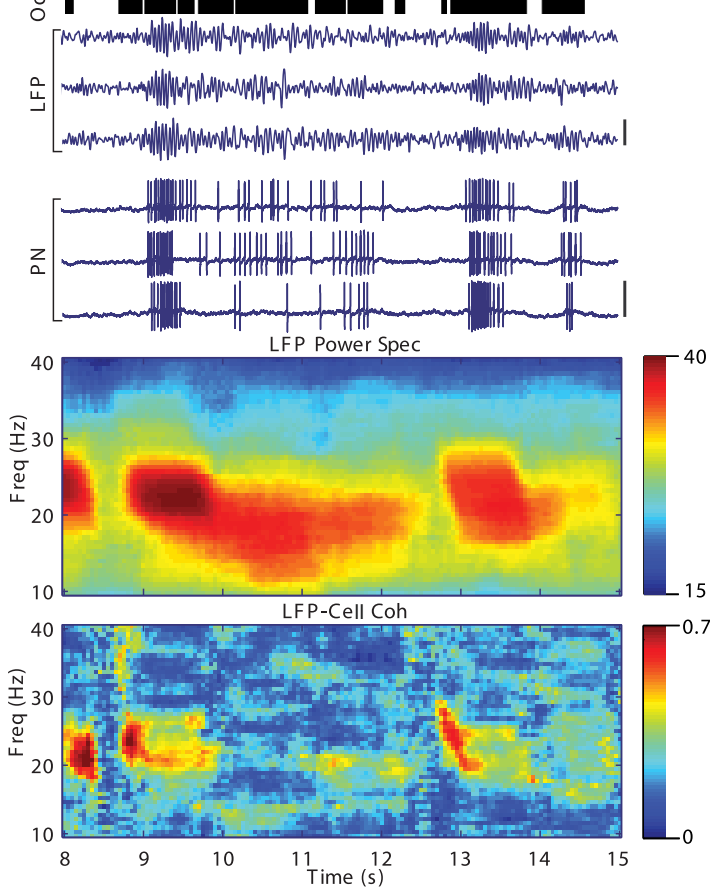

Figure 2. Dynamic odor stimulation evokes oscillatory neural activity. $\boldsymbol{a}$, Intracellular recording from an $\mathrm{LN}$ during repeated presentations of dynamic stimulus. Top, 0 dor-valve openings (black). Center, Example LN responds during three consecutive plume trials. Bottom, Trial-averaged spectrogram of the membrane potential shows prominent plume-elicited oscillatory activity (15-25 Hz). Inset, Expanded $1 \mathrm{~s}$ window shows oscillations following a brief (354 ms) valve opening. $\boldsymbol{b}$, Intracellular recording of a PN from a different animal, data as shown in $\boldsymbol{a}$. This cell was hyperpolarized to $-70 \mathrm{mV}$ by current injection to reduce spiking, highlighting subthreshold membrane potential oscillations. c, LFP ( $5-40 \mathrm{~Hz}$ bandpass filtered) from mushroom body calyx, same experiment and data format as $\boldsymbol{a}$. $\boldsymbol{d}$, Ratio of oscillatory power $(15-25 \mathrm{~Hz}$ ) in each $200 \mathrm{~ms}$ window following isolated valve openings to power in same frequency band $200 \mathrm{~ms}$ earlier. Mean $\pm 95 \%$ confidence interval, data pooled from 30 trials in each of 17 animals. $\boldsymbol{e}$, Simultaneous recordings of LFP and PN show coordinated odor-elicited oscillations in three consecutive trials. From top, odor-valve openings; LFP; membrane potential of PN; power spectra of the LFP; and a coherogram of the PN spikes with the LFP (bottom). This shows an 8 s segment extracted from the 30 s trial. PSD color units are dB/Hz. Calibration: $40 \mu \mathrm{V}$ (LFP), $20 \mathrm{mV}$ (cell).

$$
H(*)=-\sum_{*} p(*) \cdot \log _{2}[p(*)]
$$

The direct method is appropriate for our measurements because it makes minimal assumptions about the nature of the neural code. We used standard procedures for information rate calculations. To obtain an estimate of the "true" entropy rates, we tested rates for a range of different "word lengths" at bin resolutions of 5 ms precision (Strong et al., 1998) and used these measures to linearly extrapolate the entropy rates to infinite word length (Fig. $5 b$ ). The use of different precision levels ( 2 and 20 ms) for calculations of information rate did not affect the outcome. Because a limited number of stimulus repetitions can be presented during an experiment, it is necessary to apply a debiasing function to our estimates. We debiased our estimates of the various response probabilities in Equation 2 using the "Best Upper Bound" technique (Paninski, 2003). The information rates of pairs of neurons were calculated in a similar way, with the paired response distribution $r=\left(r_{1}, r_{2}\right)$ substituted into Equation 1 (Nirenberg et al., 2001).

Correlation between pairs of PNs or between individual PNs and the PN population (the local field potential, LFP) was assessed via a sliding window coherogram function. All spectral analyses used multitaper methods (Mitra and Pesaran, 1999). For coherence spectra calculated between two sets of spiking neurons we assessed significance of spectral peaks by comparison with a null hypothesis of zero coherence between two neurons with similar firing rates (Jarvis and Mitra, 2001).

Within neurons, we assessed the similarity of spike trains across trials, and across neurons, we assessed similarity of responses to the same stimulus, using a modified version of a published protocol (Padmanabhan and Urban, 2010). Briefly, for each of our 42 different cells, we binned the first 10 trials of spike train data elicited by our stimulus, and projected those binned spike trains into a reduced dimensionality principal component analysis (PCA) subspace formed by the three eigenvectors with the largest corresponding eigenvalues from decomposition of the total response covariance matrix. Then, to assess trial-to-trial variability in the responses of individual neurons we measured the Euclidean distance between individual trials and their across-trials mean, and to assess neuron-to-neuron variability we measured the distance between means of the responses of different neurons to the same stimulus.

\section{Results}

Naturalistic, dynamic olfactory stimulation regime

We wished to test how an oscillatory synchronization regime affects the capacity of neurons to transmit information about a stimulus. Information transmission can be formally expressed as 
the difference between the total entropy of the response (the capacity of the system to represent stimuli) and the stimulusconditioned entropy of the response (the amount of the total capacity actually used to represent a stimulus; Eq. 1). In principle, one could separately manipulate total and stimulus-conditioned entropies; for example, one could imagine that synchronizing pulses of inhibition could be used to selectively remove only uninformative "noise" spikes, a process that would not affect stimulus-conditioned entropy. Thus, two outcomes are possible upon removing oscillatory inhibition: the information rates of the neurons that receive inhibitory input could either stay the same or decrease, depending upon whether the inhibition balanced a reduction in the number of spiking patterns in the response (lowering the total entropy) with a reduction in the number of stimulus-conditioned spike pattern responses (lowering the conditional entropy).

To test the possible trade-offs imposed by synchrony, we needed a richly structured olfactory stimulus appropriate for the measurement of information properties. Using complex artificial plumes based on the statistical structures of temporal variability measured outdoors in real odor plumes (Fig. 1b,c; Murlis et al., 2000) allowed us to calculate lower bound estimates of information in the temporal structure of an ethologically relevant stimulus. Artificial plumes were generated by opening and closing an olfactometer's output valve, and were measured as continuous changes in odor concentration by a PID (see Materials and Methods). From the PID recordings we measured the probability that an odorant valve opening of a given duration would cause a detectable fluctuation in odorant concentration (Fig. 1f). Even odor-valve openings as brief as $30 \mathrm{~ms}$ evoked distinct voltage deflections reflecting rapid changes in odorant concentration that were highly reliable across repeated stimulus presentations (Fig. $1 d-f$ ). Net afferent activity monitored in the EAG made from the stimulated antenna closely matched changes in odorant concentration simultaneously measured near the antenna by the PID (Fig. 1d).

\section{Response of the olfactory system to the plume stimulus}

To test the ability of our stimulus to drive neural activity, we made simultaneous measurements of neural responses at several consecutive stages of processing. Like the lengthy, square pulses of odor used in prior studies (Laurent et al., 1996), the plume-like stimulus elicited oscillatory responses from the olfactory system.

Intracellular recordings made from the two populations of neurons receiving direct input from the afferents, the PNs and LNs of the antennal lobes, revealed odor-elicited $\sim 20 \mathrm{~Hz}$ sub-

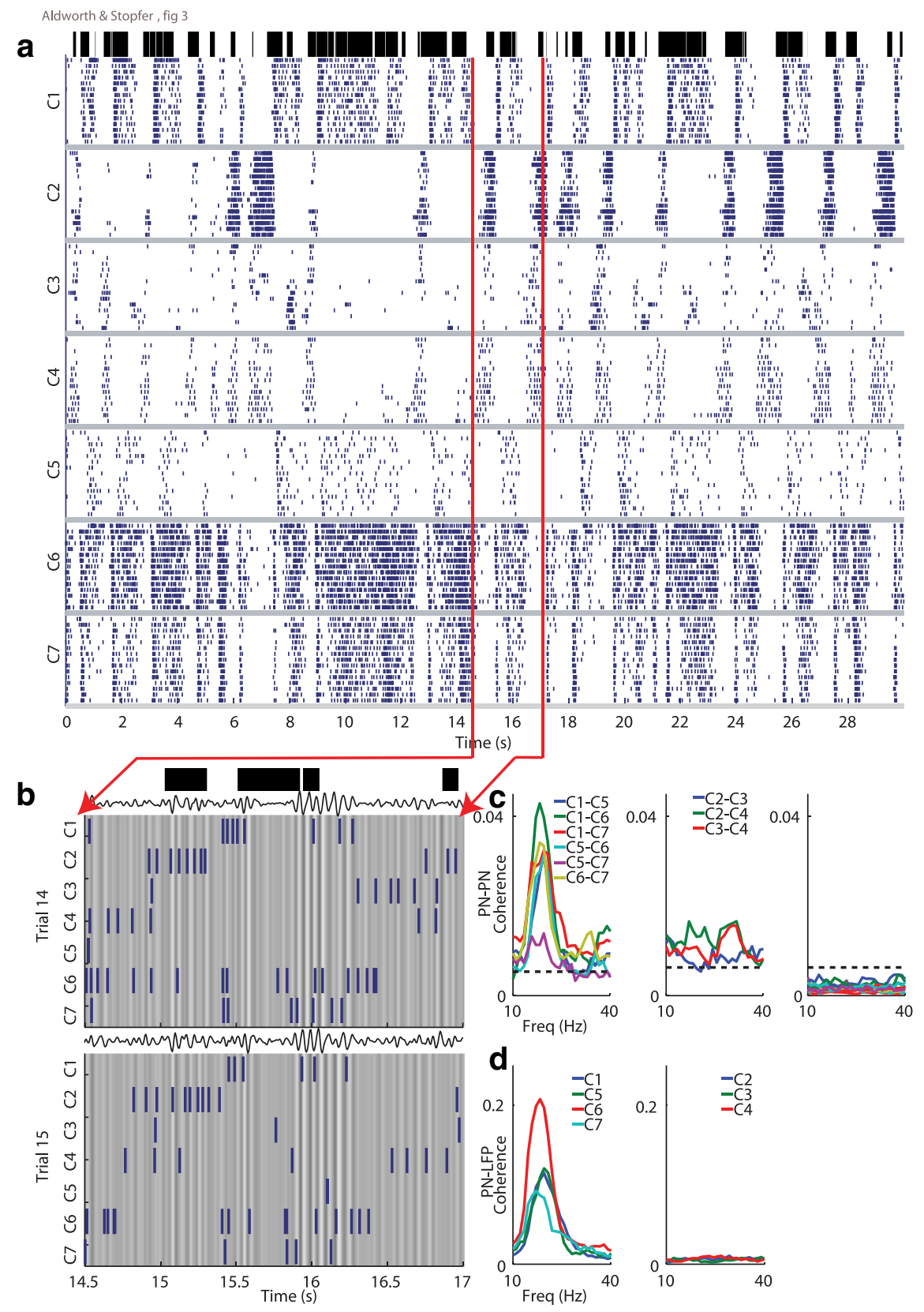

Figure 3. Plume-elicited response patterns and correlations in PNs. $\boldsymbol{a}$, Raster plot shows spiking in seven PNs simultaneously recorded by tetrodes responding to 15 dynamic stimulation presentations. PNs 1 and 5-7 tend to fire when the odor is present (when oscillations occur in the LFP), while cells $2-4$ fire when the odor is absent (when the LFP is relatively flat). Top, 0dor-valve openings. $\boldsymbol{b}$, Expanded view of trials 14 and 15 from panel $\boldsymbol{a}$, plotted with the simultaneously recorded LFP. For clarity, cycles in the LFP are plotted in grayscale superimposed with spike rasters. C, PN-PN coherence between all 21 pairings of cells, averaged over 30 s trials and 30 trials of stimulation. Left, Both PNs in the pair fired spikes during odor-valve openings. Middle, Both cells fired during odor-valve closings. Right, Mixed open-close pairings (the remaining 12 pairs, not labeled). Dashed black line in each part denotes chance-level coherence. $\boldsymbol{d}$, Coherence between PNs and the LFP. Left, With PNs that fired during odor-valve openings. Right, With PNs that fired during odor-valve closings.

threshold rhythmic oscillations in the membrane potential (Fig. $2 a, b)$. The responses of the PN population as a whole were measured near their synaptic outputs in the mushroom body by LFP recordings (Fig. 2c). Increases in LFP activity in the $15-25 \mathrm{~Hz}$ band were statistically significant even in response to odor pulses briefer than $300 \mathrm{~ms}$ (Fig. 2d), consistent with previous reports (Stopfer and Laurent, 1999). Odor-elicited bursts of spikes in individual PNs could tightly align with bursts of oscillations in the LFP (Fig. 2e). 

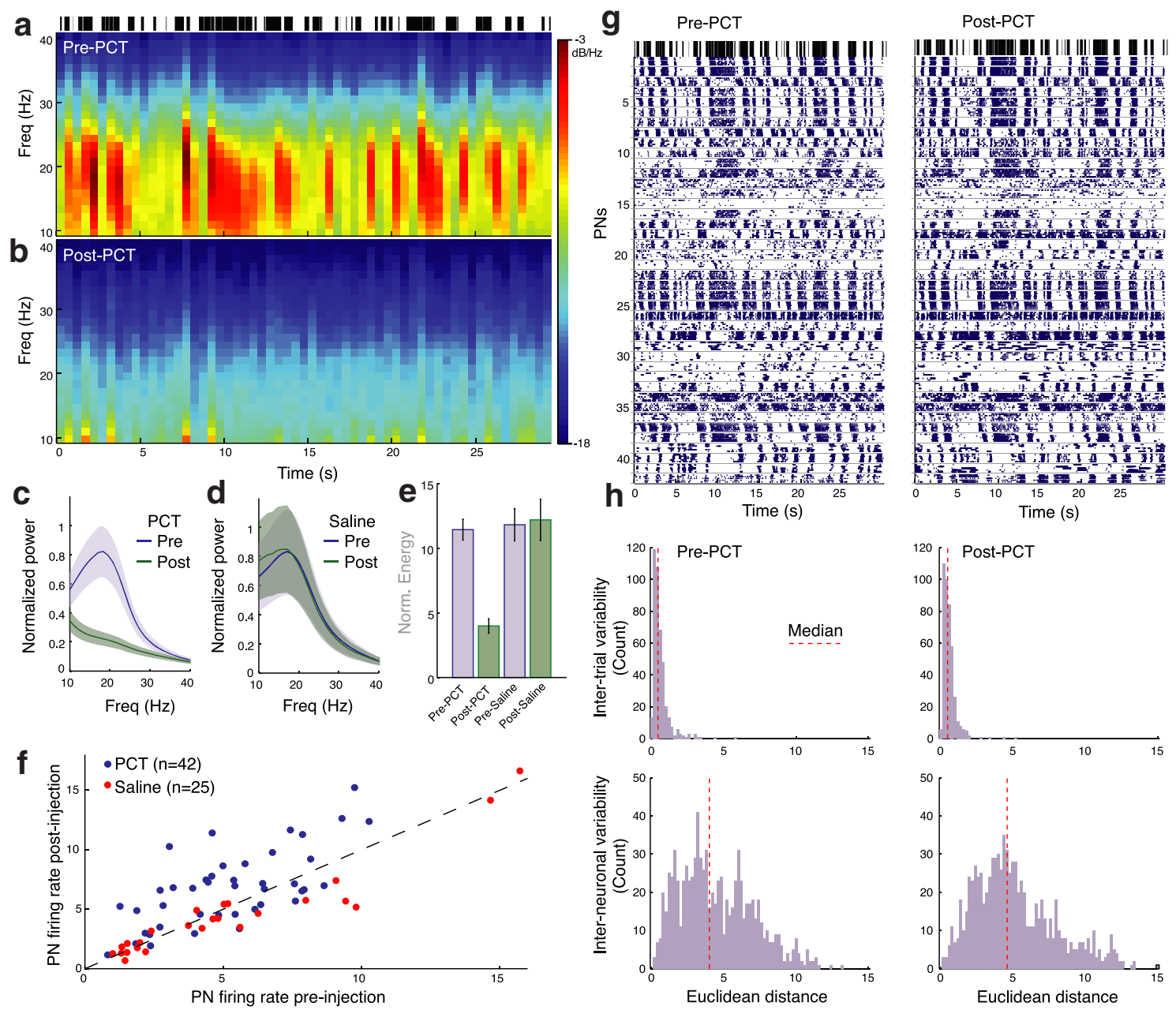

Figure 4. Focal injection of PCT abolishes oscillatory synchrony. $\boldsymbol{a}, \boldsymbol{b}$, Normalized spectrogram of plume-elicited LFP activity (mean of 19 experiments) before (a) and after (b) microinjection of PCT into the antennal lobe. PCT abolishes the $\sim 20 \mathrm{~Hz}$ oscillations. $\boldsymbol{c}$, Time-averaged mean \pm SEM power spectra $(n=19$ animals) before and after injection of PCT. $\boldsymbol{d}$, Time-averaged mean \pm SEM power spectra ( $n=8$ animals) before and after injection of saline. $\boldsymbol{e}$, Area under curve of normalized LFP spectra between $10 \mathrm{and} 30 \mathrm{~Hz}$ for pre- and post-PCT injection (left) and presaline and postsaline control injection (right). $\boldsymbol{f}$, Firing rates of PNs before and after PCT (blue) or saline (red) injection. Each point represents a single PN's firing rate averaged over time and trials. All PNs were recorded from the 19 PCT-injected or 8 saline-injected animals shown in $\boldsymbol{a}$-e. Dashed black line: identity. $\boldsymbol{g}$, Rasters show spiking responses to the first five presentations of the plume stimulus for all tested 42 PNs before (left) and after (right) injection with PCT. While abolishing oscillatory synchrony, PCT minimally altered the firing patterns of the PNs. $\boldsymbol{h}$, Histograms show Euclidean distance between individual and mean spike trains recorded from individual neurons (top) and distance between pairs of spike train means (bottom), before (left) and after (right) PCT treatment. Dashed red lines show median values.

Figure $3 a$ shows the diverse responses of seven simultaneously recorded PNs to repeated presentations of the odor plume. Although the diversity in firing patterns characteristic of PNs is most readily observed in response to relatively lengthy square pulses of odor (Laurent et al., 1996), even the brief odor pulses comprising the synthetic plume can be seen to elicit a variety of response patterning. Odor plume-elicited spikes in individual PNs reliably phase locked both with the LFP (Fig. 3b,d) and with spiking in other simultaneously recorded individual PNs (Fig. $3 c$ ). Given the very brief pulses of odor comprising the stimulus plume, some PNs responded most intensely at moments when the odor was present, while other PNs responded mainly after a pulse of odorant had been removed. In general, as expected, PNs that did not fire in the presence of the odor did not exhibit 15-25 $\mathrm{Hz}$ oscillations in spiking (Fig. $3 c, d$ ), and not all spiking during odor encounters phase locked to the LFP, in agreement with results previously reported with longer odor pulses (Laurent et al., 1996; Ito et al., 2009). Responses in any given PN varied with the presented odor: PNs that fired immediately during exposure to a plume of one odorant could initially be inhibited by a different odorant (Laurent and Davidowitz, 1994).

\section{Abolishing synchrony}

To assess the effects of oscillatory synchrony on information transfer, we blocked oscillations with localized pressure injections of the $\mathrm{GABA}_{\mathrm{a}}$ antagonist PCT into the ipsilateral antennal lobe. We found this treatment reliably desynchronized the firing of PNs during the dynamic odor stimulation (Fig. $4 a-e$ ): LFP power in the $15-25 \mathrm{~Hz}$ band of the LFP following PCT injection was significantly different ( $p=7.2 * 10^{-12}$, paired $t$ test) from pre-injection levels. In contrast, LFP power following injections of a saline control was not significantly different from preinjection power $(p=0.79$, paired $t$ test; Fig. $4 c-e)$.

PCT injections also led to significant $\left(p=8.7 * 10^{-5}\right.$, paired $t$ test) increases in the firing rates of PNs (mean increase from $5.2 \pm 0.4$ spikes/s to $6.8 \pm 0.5$ spikes/s, $N=42$ neurons in 19 animals; Fig. $4 f$ ). Firing rates increased in neurons that were active both during and after exposure to odor pulses, with no significant 
difference between the two groups ( $p=$ 0.4574, Wilcoxon rank sum test; data not shown). The increase in firing rates after PCT injection suggests odor-elicited inhibition reduces spiking in PNs.

However, PCT did not appear to affect the slower temporal structures of PN spiking elicited by the odor (Fig. 4g), which are thought to be shaped by the action of $\mathrm{GABA}_{\mathrm{b}}$-like receptors, which are not affected by PCT (MacLeod et al., 1998). To quantitatively assess the effect of PCT injection on the firing patterns of individual PNs, we compared peristimulus time histograms (PSTHs) binned at $1 \mathrm{~ms}$ precision and passed through a $2 \mathrm{~ms} \mathrm{SD}$ Gaussian filter (Chichilnisky, 2001), and examined firing patterns recorded before and after PCT injection, scaled to match the firing rates of the post-PCT injection recording. We found the RMS difference between the PSTHs to be negligible (1.31 $* 10^{-4} \pm 0.20 * 10^{-4}$ spikes $/ 10 \mathrm{~ms}$, mean \pm SE, $n=42$ neurons), indicating that PCT treatment did not change the firing patterns of the individual neurons, in agreement with previous reports (MacLeod and Laurent, 1996; MacLeod et al., 1998).

To assess variability in the responses of a given neuron over repeated trials, as well as variability in the responses of different neurons, we projected the spike trains from all neurons into a PCA subspace defined by the three most prominent eigenvectors of the joint covariance matrix, and examined the Euclidean distances between individual trials and the mean response of single neurons, as well as the distance between the mean responses of different neurons (Fig. 4h). We found no significant change in intertrial variability following PCT treatment ( $p=0.1662$, Mann-Whitney test, $n=$ 420 trial-mean pairs). However, the distance between mean spike response of different neurons increased following PCT treatment ( $p=1.352 * 10^{-5}$, Mann-Whitney test, $n=861$ ), indicating that PCT caused an increase in variability in the responses of different neurons.

\section{Assessing information rates}

To assess the effect of synchrony on information transmission, we made intracellular and extracellular recordings from PNs during dynamic stimulation, both before and after PCT application. To assess the effect of synchronous and asynchronous firing conditions we measured the mutual information between stimulus and response; this measure provides, in terms of bits, a precise and compact description of information rate, and specifies a hard limit for how much can be known about a stimulus given observations of the neural response it elicits. Under sampling conditional entropy can lead to overestimating information rates; thus, we first tested the effect of the size of the dataset on the quality of information estimation. By delivering up to 500 trials of our dynamic stimulus (Fig. $5 a$ ), we found 30 repetitions of the $30 \mathrm{~s}$ dynamic stimulus provided $<10 \%$ overestimation of the information rates (Fig. 5b,c). Because information rates measured before and after injection were both subject to the same bias, such biases should have

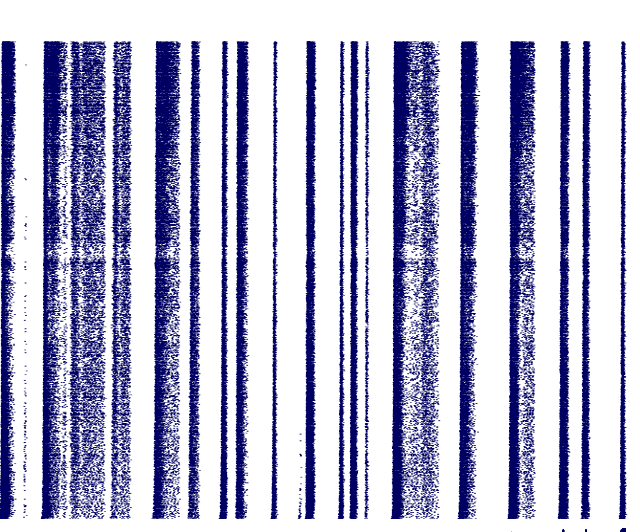

b

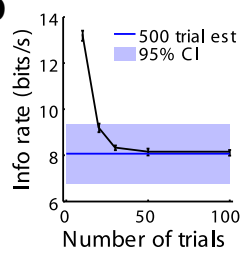

C

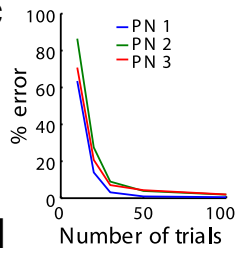

d

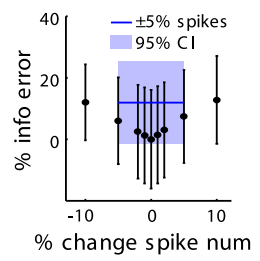

Figure 5. Information calculations given a finite dataset. $\boldsymbol{a}$, Example intracellular recording of a PN responding to 500 trials case permitted by our spike-sorting criteria (see Materials and Methods). Error bars and shaded regions represent $95 \% \mathrm{Cl}$ on regression to information of infinite length code words.

minimal impact on any net changes in rates. We established that the error rate of our spike-sorting method $(\sim 5 \%)$ fell well within a range acceptable to reach conclusions about information transmission rates (Fig. $5 d$; see Materials and Methods).

We found that PCT injection caused information rates to significantly increase (by $34.3 \pm 10.8 \%$, mean \pm SEM, $N=42$ neurons in 19 animals, $p=2.710^{-3}$; Fig. $6 a, b$ ). The increase in information rate was accompanied by significant changes in both the unconditional $(\mathrm{Hu})$ and conditional $(\mathrm{Hc})$ response entropy rates (median $\mathrm{Hu}$ pre-injection: 23.83 bits/s, median $\mathrm{Hu}$ post injection: $29.10 \mathrm{bits} / \mathrm{s}, p=0.0274$, Mann-Whitney test; median Hc pre-injection: 18.97 bits/s, median Hc post injection: 21.74 bits/s, $p=0.0321$, Mann-Whitney test). In contrast, when a control injection of saline was substituted for PCT, the information rates decreased by $7.7 \pm 2.7 \%$ (mean $\pm \mathrm{SE}, n=25$ neurons in eight animals, $p=9.6 * 10^{-3}$; Fig. $6 b$ ), likely from nonspecific effects of the injection procedure, or the passage of time. These decreases in information rates were accompanied by small and nonsignificant decreases in entropy rates (median Hu pre: 20.40 bits/s, median Hu post: 18.05 bits/s, $p=0.5737$, Mann-Whitney test; median Hc pre: 16.78 bits/s, median Hc post: 15.14 bits/s, $p=0.5869$, Mann-Whitney test). The difference in effect on information rates between PCT and control treatments was highly significant (Fig. $6 b ; p=1.2 * 10^{-3}$ ) and was the same for responses during (on) or after (off) odor pulses (Fig. 6c). This result suggests odor-elicited periodic GABAergic inhibition reduces a $\mathrm{PN}^{\prime}$ s ability to transmit information.

Information about odorants is not only carried by individual PNs, but is also distributed across ensembles of PNs (Laurent et 

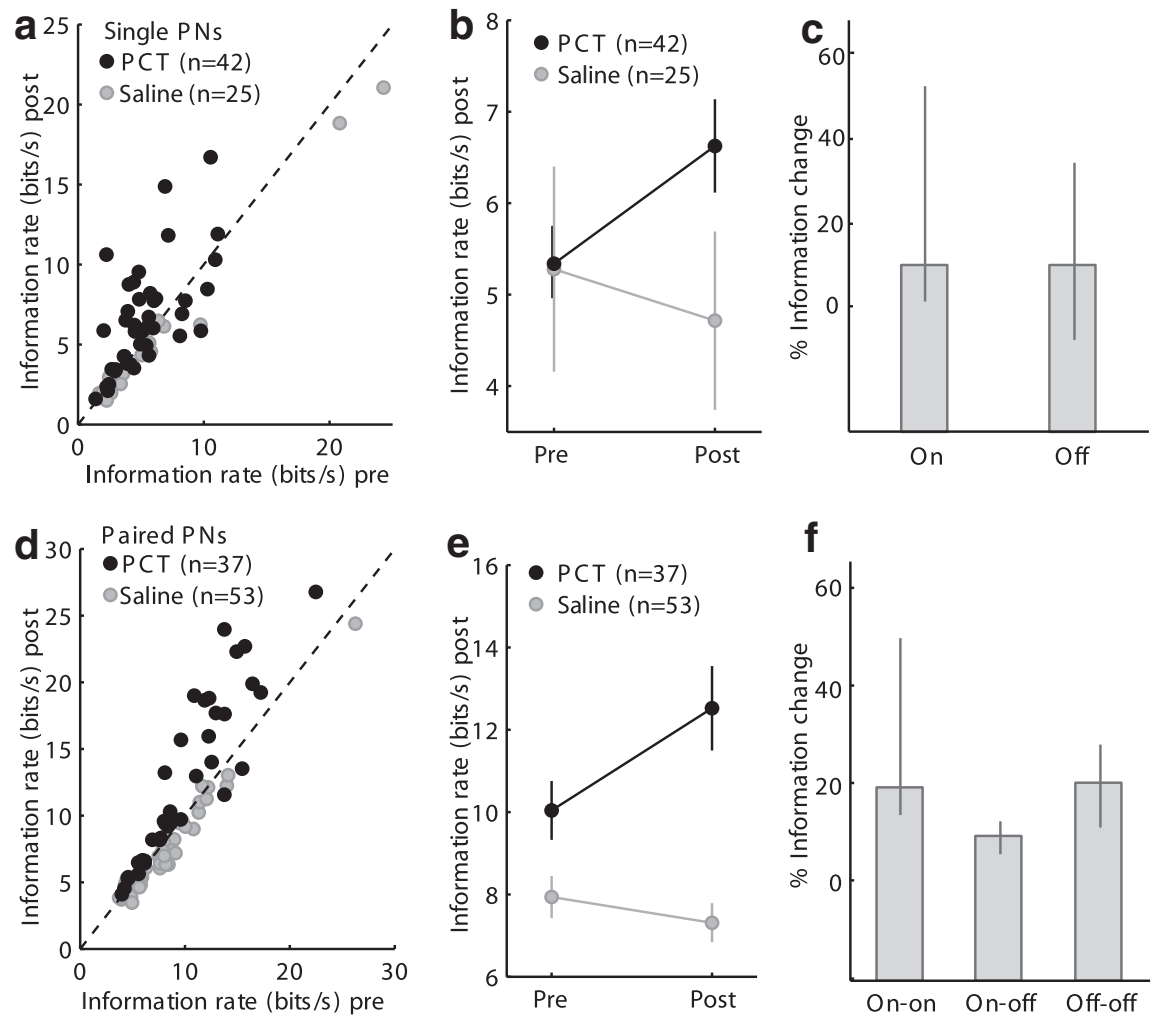

Figure 6. Desynchronization reduces information transmission rates in PNs. $\boldsymbol{a}$, Information rates transmitted by single PNs about plume-like stimuli before and after PCT (black) or saline (gray) injection; PNs are same as shown in Figure 4f. Dashed line: identity. $\boldsymbol{b}$, Population means \pm SEM from data in $\boldsymbol{a}$. $\boldsymbol{c}$, Percentage change (median \pm interquartile range) in information rates following PCT injection for single PNs, grouped by whether they most often fired during odorant-valve openings ("on") or during valve closings ("off"), were not significantly different (Mann-Whitney test, $p=0.44$ ). $\boldsymbol{d}$, Information rates transmitted by pairs of PNs about plume-like stimuli before and after PCT (black) or saline (gray) injection. $\boldsymbol{e}$, Population means \pm SEM from data in $\boldsymbol{d}$. $\boldsymbol{f}$, Percentage change in information rates for pairs of PNs following PCT injection. PN pairs were divided into three groups depending on whether both neurons in the pair fired during odorant-valve opening ("on") or during valve closings ("off"). Following PCT injection, "on-off" pairings significantly (Kruskal-Wallis test, $p=0.03$ ) increased information rates less than "on-on" or "off-off" pairings.

al., 1996; Stopfer et al., 2003; Mazor and Laurent, 2005). To test the effect of oscillatory synchrony on information distributed across small ensembles of neurons, we repeated the information rate calculations using the responses of simultaneously recorded pairs of neurons. We found that PCT application significantly increased the information rate between the stimulus and paired neuronal responses (by $22.3 \pm 3.7 \%$, mean \pm SE, $n=37$ pairs of neurons in 11 animals, $p=9.3 * 10^{-6}$; Fig. $\left.6 d, e\right)$. The increase in information rates in pairs of cells was accompanied by increases in the underlying entropy rates (median $\mathrm{Hu}$ pre: $40.91 \mathrm{bits} / \mathrm{s}$, median $\mathrm{Hu}$ post: 50.14 bits/s, $p=0.0155$, Mann-Whitney test; median Hc pre: 31.17 bits/s, median Hc post: 38.26 bits/s, $p=$ 0.0083 , Mann-Whitney test). When saline was injected instead of PCT, the information rate between stimulus and paired neuronal response decreased significantly by $7.2 \pm 1.3 \%$ (mean \pm SEM, $n=53$ pairs neurons from four animals, $\left.p=6.6 * 10^{-8}\right)$. The decrease in information between saline-treated pairs of cells was accompanied by small, nonsignificant decreases in the underlying entropy rates (median Hu pre: 33.75 bits/s, median Hu post: $32.88 \mathrm{bits} / \mathrm{s}, p=0.2607$, Mann-Whitney test; median Hc pre: 26.27 bits/s, median Hu post: 25.43 bits/s, $p=0.3764$, MannWhitney test). The difference in effect between PCT and control treatments for the paired neuronal recordings was highly significant $\left(p=6.8 * 10^{-11}\right.$; Fig. $\left.6 e\right)$, and significantly varied with the way PNs in a pair responded to an odor pulse, with “on-off” pairings increasing in information rate by less than both "onon" and "off-off" pairings (Fig. 6f; Kruskal-Wallace test, $p=0.0301)$.

We had observed that firing rates of neurons increased following PCT application (Fig. 4f). To examine the relationship between firing rate and information transmission rate, we calculated the correlation coefficient between the change in firing rate (pretreatment vs post treatment) and the change in information transmission rate. For both single PNs, and pairs of PNs (firing rates summed), the correlation was highly significant $\left(R^{2}=0.6362, p=6.0 * 10^{-6}\right.$ and $R^{2}=$ $0.7460, p=1.0 * 10^{-7}$, respectively). To further examine whether factors other than firing rate (such as the temporal precision of spiking) might contribute to changes in information rate, we calculated changes in information efficiency as bits per spike before and after PCT application. In neither single nor paired recordings of PNs did the change in information efficiency differ significantly between PCT and saline control conditions $(p=$ 0.4161 and $p=0.0543$, nonpaired $t$ tests, respectively). This result agrees with our earlier finding that, although trial-to-trial variability within a single neuron does not significantly change following PCT treatment, the overall discriminability between mean firing patterns of different neurons increases because the spike rate increases. These results show that odorelicited periodic GABAergic inhibition reduces information rates in $\mathrm{PNs}$ by reducing the number of odor-elicited spikes they can fire.

\section{Discussion}

We examined the relationship between oscillatory synchrony and information transmission in the olfactory system. Combining physiological recordings, pharmacological treatments, and information theoretic analyses, we found that information about the timing of a stimulus transmitted by PNs is reduced by the GABAergic inhibition that underlies oscillatory synchrony in the early processing layers of the insect olfactory system. Additionally, we found that this reduction in information transmission rate is caused by inhibition-induced reductions in the firing rates of the PNs, rather than by changes in the average amount of information carried by each action potential. Because PNs provide the only pathway for olfactory signals to higher processing stages (including those involved in the formation of memories and motor output), their firing patterns present a fundamental limit to the animal's ability to process signals from the environment. The inhibition-induced reduction in transmission capacity we observed in the olfactory system is likely to occur in any neural circuit using periodic inhibition for sensory processing.

When responding to pulses of odor, PNs become organized into odorant-specific ensembles, their spikes synchronized by oscillating waves of inhibition from LNs (Laurent and Davidowitz, 
1994; Stopfer et al., 1997; Ito et al., 2009; Tanaka et al., 2009). The temporal patterning of this activity has been shown to contribute to the highly reliable (Bazhenov et al., 2005) and sparse responses of Kenyon cells, neurons in the mushroom body postsynaptic to PNs. Kenyon cells remain silent unless their high firing thresholds (and inhibitory feedback) are exceeded by coactive, convergent excitatory input from many presynaptic PNs (Perez-Orive et al., 2002, 2004; Jortner et al., 2007). Periodic inhibition is thought to play an important role in organizing the coordinated excitation needed to drive Kenyon cells to spike. The oscillatory firing patterns established in the antennal lobe are maintained and transmitted throughout the olfactory system as a result of specific mechanisms, such as spike timing-dependent plasticity (Cassenaer and Laurent, 2007), which ensure the fidelity of the oscillatory signals. Indeed, the ability of neurons postsynaptic to the Kenyon cells (e.g., $\beta$-lobe neurons) to discriminate between different odorants is compromised when oscillations are abolished pharmacologically (MacLeod et al., 1998). In addition, at the behavioral level, insects show impaired performance of fine odorant discrimination tasks when oscillations are abolished (Stopfer et al., 1997). Thus, the odor-induced oscillations generated in the antennal lobe contribute to an insect's ability to discriminate between different odors.

In a variety of species, relatively lengthy pulses of diverse odors have been shown to elicit oscillatory synchronization, but whether oscillatory mechanisms are evoked by the temporally dynamic, plume-like stimuli used by insects to track point sources has been called into question (Vickers et al., 2001). Here we show that the oscillation machinery is engaged even by staccato dynamic stimuli with the statistical structure of odorant plumes; indeed, the oscillatory activity itself follows the temporal dynamics of the stimulus. Our result suggests that the principles of olfactory signal processing first demonstrated using slowly changing stimuli are also relevant to a wide range of environmental conditions (Stopfer et al., 2003; Brown et al., 2005; Broome et al., 2006).

Several previous studies have examined the extent to which it is necessary to know the correlation structure among all the neurons in a population to estimate the information those neurons can convey about the stimulus (Meister et al., 1995; Nirenberg et al., 2001; Schneidman et al., 2003, 2011; Averbeck and Lee, 2004; Latham and Nirenberg, 2005; Pillow et al., 2008). Here, we asked whether the neural mechanisms used to generate correlations also affect the amount of information that can be transmitted about the stimulus. Our results show that the amount of information about the temporal structure of a stimulus that can be encoded is reduced by the inhibition used to establish oscillatory synchrony.

The temporal dynamics of an odor plume carries information important to animals, as it reflects both the distance from the plume's source as well as the air viscosity over the intervening terrain (Murlis et al., 2000). Some insects, mosquitos, for example, are specifically attracted to plume-like presentations of odors from host animals (Geier et al., 1999), and recent work has shown that frequency and intermittency of filaments within odor plumes can have a strong effect on an insect's ability to locate the odor source (Riffell et al., 2014). Our repeatable olfactory stimulus was designed to reflect the rapid fluctuations observed in naturally occurring plumes. Our results show that the insect olfactory system has abundant capacity to encode the information contained in these fluctuations. The information content (entropy rate) of the two independent gamma distributions (Fig $1 b, c)$ used to control odor-valve openings (assuming indepen- dence between the two) was analytically determined to be 6.5 bits/s, less than the 8 bits/s that the average pair of neurons in our dataset could encode about stimulus dynamics (Fig. 6e). Flying insects interact with the temporal dynamics of odor plumes by engaging in sideways casting movements through the odor plume during flight (Rutkowski et al., 2009). The temporal frequencies of such interactions are relatively low compared with the dynamics of the plume itself, so the combined interactions should have an information content of less than double the 6.5 bits/s of the odor plume alone, and substantially less than the highest information rate of our recorded pairs of neurons ( $>20 \mathrm{bits} / \mathrm{s})$. Additionally, most insects have at least several hundred PNs in each antenna lobe (Galizia and Rössler, 2010); because the information rate encoded within a population of PNs scales with the size of the population (Geffen et al., 2009), the PN population should offer substantial excess capacity to encode stimulus dynamics beyond what insects encounter in the natural environment. In the locust, previous experimental recordings from the Kenyon cells and $\beta$-lobe neurons of the mushroom body have shown that discriminating odor identity requires decoding input pooled from large portions of the PN population (MacLeod et al., 1998; Perez-Orive et al., 2004).

The olfactory world contains information not only about the temporal dynamics of an odor, but also about the odor's chemical identity and concentration. Here, we focused on stimulus timing because we were able to provide, with our synthetic odor plume, a broad sample of an environmentally meaningful stimulus space. Because relatively little is known of the statistics of the natural world of odorants and their concentrations, and because this stimulus space is very large, it is difficult to analyze with the tools of information theory. Clearly, a portion of the coding capacity of PNs is used to carry such information (Stopfer et al., 1997, 2003; MacLeod et al., 1998; Perez-Orive et al., 2002, 2004; Brown et al., 2005; Mazor and Laurent, 2005; Broome et al., 2006).

Our work shows that the oscillatory synchronization of olfactory PNs through rhythmic inhibition reduces the capacity of these neurons to transmit information about the temporal structure of an odor stimulus. Oscillatory synchronization, however, has been suggested to serve a variety of information-processing functions, including reformatting to sparse, easily distinguished and memorized representations of odors (Laurent, 2002). Our results suggest this reformatting comes at a cost, and represents a fundamental trade-off between coding capacity and other aspects of format utility.

\section{References}

Averbeck BB, Lee D (2004) Coding and transmission of information by neural ensembles. Trends Neurosci 27:225-230. CrossRef Medline

Bazhenov M, Stopfer M, Sejnowski TJ, Laurent G (2005) Fast odor learning improves reliability of odor responses in the locust antennal lobe. Neuron 46:483-492. CrossRef Medline

Broome BM, Jayaraman V, Laurent G (2006) Encoding and decoding of overlapping odor sequences. Neuron 51:467-482. CrossRef Medline

Brown SL, Joseph J, Stopfer M (2005) Encoding a temporally structured stimulus with a temporally structured neural representation. Nat Neurosci 8:1568-1576. CrossRef Medline

Cassenaer S, Laurent G (2007) Hebbian STDP in mushroom bodies facilitates the synchronous flow of olfactory information in locusts. Nature 448:709-713. CrossRef Medline

Chichilnisky EJ (2001) A simple white noise analysis of neuronal light responses. Network 12:199-213. CrossRef Medline

Galizia CG, Rössler W (2010) Parallel olfactory systems in insects: anatomy and function. Annu Rev Entomol 55:399-420. CrossRef Medline

Geffen MN, Broome BM, Laurent G, Meister M (2009) Neural encoding of rapidly fluctuating odors. Neuron 61:570-586. CrossRef Medline 
Geier M, Bosch OJ, Boeckh J (1999) Influence of odour plume structure on upwind flight of mosquitoes towards hosts. J Exp Biol 202:1639-1648. Medline

Gray CM (1999) The temporal correlation hypothesis of visual feature integration: still alive and well. Neuron 24:31-47, 111-125. CrossRef Medline

Gupta N, Stopfer M (2012) Functional analysis of a higher olfactory center, the lateral horn. J Neurosci 32:8138-8148. CrossRef Medline

Hill DN, Mehta SB, Kleinfeld D (2011) Quality Metrics to Accompany Spike Sorting of Extracellular Signals. J Neurosci 31:8699-8705. CrossRef Medline

Hosler JS, Buxton KL, Smith BH (2000) Impairment of olfactory discrimination by blockade of GABA and nitric oxide activity in the honey bee antennal lobes. Behav Neurosci 114:514-525. CrossRef Medline

Ito I, Bazhenov M, Ong RC, Raman B, Stopfer M (2009) Frequency transitions in odor-evoked neural oscillations. Neuron 64:692-706. CrossRef Medline

Jarvis MR, Mitra PP (2001) Sampling properties of the spectrum and coherency of sequences of action potentials. Neural Comput 13:717-749. CrossRef Medline

Jortner RA, Farivar SS, Laurent G (2007) A simple connectivity scheme for sparse coding in an olfactory system. J Neurosci 27:1659-1669. CrossRef Medline

Latham PE, Nirenberg S (2005) Synergy, redundancy, and independence in population codes, revisited. J Neurosci 25:5195-5206. CrossRef Medline

Laurent G (2002) Olfactory network dynamics and the coding of multidimensional signals. Nat Rev Neurosci 3:884-895. CrossRef Medline

Laurent G, Davidowitz H (1994) Encoding of olfactory information with oscillating neural assemblies. Science 265:1872-1875. CrossRef Medline

Laurent G, Seymour-Laurent KJ, Johnson K (1993) Dendritic excitability and a voltage-gated calcium current in locust nonspiking local interneurons. J Neurophysiol 69:1484-1498. Medline

Laurent G, Wehr M, Davidowitz H (1996) Temporal representations of odors in an olfactory network. J Neurosci 16:3837-3847. Medline

MacLeod K, Laurent G (1996) Distinct mechanisms for synchronization and temporal patterning of odor-encoding neural assemblies. Science 274:976-979. CrossRef Medline

MacLeod K, Bäcker A, Laurent G (1998) Who reads temporal information contained across synchronized and oscillatory spike trains? Nature 395: 693-698. CrossRef Medline

Mazor O, Laurent G (2005) Transient dynamics versus fixed points in odor representations by locust antennal lobe projection neurons. Neuron 48 : 661-673. CrossRef Medline

Meister M, Lagnado L, Baylor DA (1995) Concerted signaling by retinal ganglion cells. Science 270:1207-1210. CrossRef Medline

Mitra PP, Pesaran B (1999) Analysis of dynamic brain imaging data. Biophys J 76:691-708. CrossRef Medline

Murlis J, Willis MA, Cardé RT (2000) Spatial and temporal structures of pheromone plumes in fields and forests. Physiol Entomol 25:211-222. CrossRef

Nirenberg S, Carcieri SM, Jacobs AL, Latham PE (2001) Retinal ganglion cells act largely as independent encoders. Nature 411:698-701. CrossRef Medline

Padmanabhan K, Urban NN (2010) Intrinsic biophysical diversity decorrelates neuronal firing while increasing information content. Nat Neurosci 13:1276-1282. CrossRef Medline
Paninski L (2003) Estimation of entropy and mutual information. Neural Comput 15:1191-1253. CrossRef

Perez-Orive J, Mazor O, Turner GC, Cassenaer S, Wilson RI, Laurent G (2002) Oscillations and sparsening of odor representations in the mushroom body. Science 297:359-365. CrossRef Medline

Perez-Orive J, Bazhenov M, Laurent G (2004) Intrinsic and circuit properties favor coincidence detection for decoding oscillatory input. J Neurosci 24:6037-6047. CrossRef Medline

Pillow JW, Shlens J, Paninski L, Sher A, Litke AM, Chichilnisky EJ, Simoncelli EP (2008) Spatio-temporal correlations and visual signalling in a complete neuronal population. Nature 454:995-999. CrossRef Medline

Pouzat C, Mazor O, Laurent G (2002) Using noise signature to optimize spike-sorting and to assess neuronal classification quality. J Neurosci Methods 122:43-57. CrossRef Medline

Reisenman CE, Dacks AM, Hildebrand JG (2011) Local interneuron diversity in the primary olfactory center of the moth Manduca sexta. J Comp Physiol A Neuroethol Sens Neural Behav Physiol 197:653-665. CrossRef Medline

Riffell JA, Shlizerman E, Sanders E, Abrell L, Medina B, Hinterwirth AJ, Kutz JN (2014) Sensory biology. Flower discrimination by pollinators in a dynamic chemical environment. Science 344:1515-1518. CrossRef Medline

Rutkowski AJ, Quinn RD, Willis MA (2009) Three-dimensional characterization of the wind-borne pheromone tracking behavior of male hawkmoths, Manduca sexta. J Comp Physiol A Neuroethol Sens Neural Behav Physiol 195:39-54. CrossRef Medline

Schneidman E, Bialek W, Berry MJ 2nd (2003) Synergy, redundancy, and independence in population codes. J Neurosci 23:11539-11553. Medline

Schneidman E, Puchalla JL, Segev R, Harris RA, Bialek W, Berry, MJ 2nd (2011) Synergy from silence in a combinatorial neural code. J Neurosci 31:15732-15741. CrossRef Medline

Seki Y, Rybak J, Wicher D, Sachse S, Hansson BS (2010) Physiological and morphological characterization of local interneurons in the Drosophila antennal lobe. J Neurophysiol 104:1007-1019. CrossRef Medline

Shannon CE (1948) A mathematical theory of communication. Bell Syst Tech J 27:379-423. CrossRef

Singer W (1999) Neuronal synchrony: a versatile code for the definition of relations? Neuron 24:49-65, 111-125. CrossRef Medline

Stopfer M, Laurent G (1999) Short-term memory in olfactory network dynamics. Nature 402:664-668. CrossRef Medline

Stopfer M, Bhagavan S, Smith BH, Laurent G (1997) Impaired odour discrimination on desynchronization of odour-encoding neural assemblies. Nature 390:70-74. CrossRef Medline

Stopfer M, Jayaraman V, Laurent G (2003) Intensity versus identity coding in an olfactory system. Neuron 39:991-1004. CrossRef Medline

Strong SP, Koberle R, de Ruyter van Steveninck RR, Bialek W (1998) Entropy and information in neural spike trains. Phys Rev Lett 80:197-200. CrossRef

Tanaka NK, Ito K, Stopfer M (2009) Odor-evoked neural oscillations in Drosophila are mediated by widely branching interneurons. J Neurosci 29:8595-8603. CrossRef Medline

Vickers NJ, Christensen TA, Baker TC, Hildebrand JG (2001) Odour-plume dynamics influence the brain's olfactory code. Nature 410:466-470. CrossRef Medline 